\title{
$\beta$-Lactamase-Producing Multidrug-Resistant Bacterial Pathogens from Tracheal Aspirates of Intensive Care Unit Patients at National Institute of Neurological and Allied Sciences, Nepal
}

\author{
Santosh Khanal, ${ }^{1,2}$ Dev Raj Joshi, ${ }^{1}$ Dwij Raj Bhatta, ${ }^{1}$ \\ Upendra Devkota, ${ }^{3}$ and Bharat Mani Pokhrel ${ }^{4}$ \\ ${ }^{1}$ Central Department of Microbiology, Tribhuvan University, Kathmandu, Nepal \\ ${ }^{2}$ National College (NIST), Tribhuvan University, Kathmandu, Nepal \\ ${ }^{3}$ National Institute of Neurological and Allied Sciences (NINAS), Kathmandu, Nepal \\ ${ }^{4}$ Department of Microbiology, Institute of Medicine (IOM), Tribhuvan University, Kathmandu, Nepal \\ Correspondence should be addressed to Santosh Khanal; santoshkhanal007@gmail.com
}

Received 24 June 2013; Accepted 22 July 2013

Academic Editors: G. Alexandre, H. Asakura, and I. Morozov

Copyright (c) 2013 Santosh Khanal et al. This is an open access article distributed under the Creative Commons Attribution License, which permits unrestricted use, distribution, and reproduction in any medium, provided the original work is properly cited.

\begin{abstract}
The widespread use of tracheal intubation and mechanical ventilation to support the critically ill patients increases the risk of development of tracheobronchitis and bronchopneumonia. This cross-sectional study was conducted with an aim to isolate and identify bacterial pathogens from tracheal aspirates producing extended-spectrum $\beta$-lactamase (ESBL), AmpC $\beta$-lactamase, and metallo- $\beta$-lactamase (MBL) from August 2011 to April 2012 at National Institute of Neurological and Allied Sciences (NINAS), Kathmandu, Nepal. ESBL was detected by combined disk assay using cefotaxime and cefotaxime with clavulanate, AmpC $\beta$ lactamase by inhibitor-based method using cefoxitin and phenylboronic acid, and MBL by Imipenem-EDTA combined disk method. 167 bacterial strains were isolated from 187 samples and majority of them were Acinetobacter spp. followed by Klebsiella pneumoniae with $32.9 \%$ and $25.1 \%$, respectively. $68.8 \%$ of isolates were multidrug resistant (MDR) and Acinetobacter spp. constituted 85.4\%. ESBL, AmpC $\beta$-lactamase, and MBL were detected in 35 (25\%), 51 (37.2\%), and 11 (36.7\%) isolates, respectively. Pseudomonas spp. (42.8\%) were the predominant ESBL producer while Acinetobacter spp. were the major AmpC $\beta$-lactamase producer (43.1\%) and MBL producer (54.5\%).
\end{abstract}

\section{Introduction}

Tracheostomy is a surgical procedure that creates an opening directly into the trachea to ventilate and aspirate the patient in critical care setting [1]. The incidence of ventilator-associated pneumonia (VAP) ranges from 10 to $25 \%$ of all intensive care unit (ICU) patients resulting in high mortality rate of $22-71 \%$, which is 6-21 times higher in intubated patients [2].

The tracheostomized patients are colonized or infected with bacteria either endogenously or exogenously. Exogenous bacteria include Pseudomonas spp., Acinetobacter spp., methicillin-resistant Staphylococcus aureus (MRSA), and members of Enterobacteriaceae and endogenous bacteria include Streptococcus pneumoniae, Haemophilus influen$z a e$, and Moraxella catarrhalis. These bacteria are usually resistant to multiple antibiotics and cause either tracheobronchitis or bronchopneumonia [3]. Risk factors for colonization or infection with multidrug-resistant bacterial species include prolonged length of hospital stay, exposure to an ICU, receipt of mechanical ventilation, colonization pressure, exposure to broad-spectrum antimicrobial agents, recent surgery, invasive procedures, and underlying severity of illness $[4,5]$. 
$\beta$-Lactamases are the commonest cause of bacterial resistance to $\beta$-lactam antimicrobial agents, which are used in the treatment of various serious infections. With the increased use of antimicrobial agents, bacteria responded with a variety of new $\beta$-lactamases including extended-spectrum $\beta$-lactamases, plasmid-mediated AmpC $\beta$-lactamases and metallo$\beta$-lactamases [6]. Infections caused by multidrug-resistant bacteria expressing $\beta$-lactamases pose serious challenges to clinicians because these bacteria are resistant to a broad range of $\beta$-lactams, including third-generation cephalosporins, and nosocomial infections caused by these organisms complicate therapy and limit treatment options $[6,7]$.

The emergence and spread of antimicrobial resistance due to the production of $\beta$-lactamases as a major problem have drawn attention to a need for better diagnostic techniques and newer drugs to allow more specific therapy. Therefore, the characterization and antibiotic susceptibility pattern of $\beta$-lactamase-producing organisms can lead to successful infection control, involving antimicrobial stewardship and public health interventions aimed at controlling the emergence of such life-threatening multidrug-resistant bacteria. Hence, this study was undertaken to detect the bacterial pathogens and determine the antimicrobial resistance pattern of clinically relevant bacteria producing extended-spectrum $\beta$-lactamase, AmpC $\beta$-lactamase, and metallo- $\beta$-lactamase from tracheal aspirate of patients admitted to ICU.

\section{Materials and Methods}

This cross-sectional study was conducted at National Institute of Neurological and Allied Sciences, Bansbari, Kathmandu, Nepal, from August 2011 to April 2012. A total of 187 tracheal aspirate samples were included in the study.

2.1. Specimen Collection. The samples were collected in mucus trapper by applying negative pressure through automated machine by experienced physician and samples were immediately transported to the laboratory.

2.2. Culture of the Specimen. The specimens were inoculated on blood agar, MacConkey agar, and chocolate agar plates. In the chocolate agar plate, a $5 \mu \mathrm{g}$ optochin disc and a $10 \mathrm{U}$ bacitracin disc were added to screen out $S$. pneumoniae and $H$. influenzae, respectively, and the plates were incubated at $37^{\circ} \mathrm{C}$ overnight in candle jar, whereas, the MacConkey and blood agar plates were incubated under aerobic condition [8].

2.3. Identification and Antibiotic Susceptibility Test. The isolates were identified on the basis of colony characterization, staining, and biochemical tests such as oxidase, catalase, sulfide indole motility, citrate, urea hydrolysis, triple sugar iron agar test, and coagulase tests [8]. Antibiotic sensitivity test was performed using the Kirby-Bauer disk diffusion method and sensitivity results were interpreted according to CLSI guidelines [9]. Multidrug resistance was defined as resistance to three or more of the antimicrobial agents belonging to different structural classes [10].
TABLE 1: Pattern of microbial isolates from tracheal aspirate of ICU patients.

\begin{tabular}{lcc}
\hline Organisms & Frequency & MDR (\%) \\
\hline Gram-negative bacteria & $\mathbf{1 5 4}$ & $\mathbf{1 0 8}(\mathbf{7 0 . 1})$ \\
Acinetobacter spp. & 55 & $47(85.4)$ \\
K. pneumoniae & 42 & $31(73.8)$ \\
Pseudomonas spp. & 37 & $19(51.3)$ \\
E. coli & 12 & $6(50)$ \\
Enterobacter spp. & 3 & $2(66.7)$ \\
K. oxytoca & 2 & $2(100)$ \\
Citrobacter freundii & 2 & 0 \\
Proteus vulgaris & 1 & $1(100)$ \\
Gram-positive bacteria & $\mathbf{1 3}$ & $\mathbf{7 ( 5 3 . 8 )}$ \\
S. aureus & 12 & $6(50)$ \\
S. pneumoniae & 1 & $1(100)$ \\
Total & $\mathbf{1 6 7}$ & $\mathbf{1 1 5}(\mathbf{6 8 . 8})$ \\
\hline
\end{tabular}

2.4. Test for ESBL, AmpC $\beta$-Lactamase, and MBL Production. ESBL was detected by combined disk assay using cefotaxime $(30 \mu \mathrm{g})$ and cefotaxime $(30 \mu \mathrm{g})$ with clavulanate $(10 \mu \mathrm{g})$ [9], AmpC $\beta$-lactamase by inhibitor-based method using cefoxitin $(30 \mu \mathrm{g})$ and cefoxitin $(30 \mu \mathrm{g})$ with phenylboronic acid $(20 \mu \mathrm{L})$ [11], and MBL by combined disk assay using Imipenem $(10 \mu \mathrm{g})$ and Imipenem $(10 \mu \mathrm{g})$ with $100 \mathrm{mM}$ EDTA $(10 \mu \mathrm{L})[12]$.

\section{Results}

Out of 187 tracheal aspirate samples, 138 males and 49 females, 146 (78.1\%) samples showed significant growth with 21 polymicrobial growth. 167 bacterial strains were identified and among them, 115 (68.8\%) were multidrug resistant. Among 167 isolates, Gram-negative bacteria constituted 154 (92.2\%) of the total isolates, among which 108 (70.1\%) were MDR. Among Gram-negatives, Acinetobacter spp. were the most frequently isolated species with $55(32.9 \%)$ isolates and among them, 47 (85.4\%) were found to be MDR-strains. Gram-positive organisms constituted $13(7.8 \%)$ of the total isolates and 7 (53.8\%) of them were MDR. Staphylococcus aureus constituted 12 isolates and 6 (50\%) of these were MDR. The results are shown in Table 1.

Acinetobacter spp. showed high rate of resistance to cefepime (96.4\%), cotrimoxazole $(96.4 \%)$, cefoxitin $(94.5 \%)$, cefotaxime $(87.3 \%)$, gentamicin $(83.6 \%)$, and ciprofloxacin (80\%). Similarly, high rate of resistance was observed among K. pneumoniae and Pseudomonas spp. to cotrimoxazole, cefoxitin, cefotaxime, cefepime, and ciprofloxacin. The results are shown in Table 2.

Staphylococcus aureus showed high rate of resistance to ampicillin (83.3\%), cotrimoxazole (50\%), erythromycin (41.7\%), cloxacillin (33.3\%), and ciprofloxacin (33.3\%). The results are shown in Table 3.

ESBL production was confirmed in $35(25 \%)$ isolates and the majority consisted of Pseudomonas spp. with 15 (42.8\%) followed by K. pneumoniae with 12 (34.3\%). Out of the 51 (37.2\%) AmpC $\beta$-lactamase-positive isolates, Acinetobacter 
TABle 2: Antibiotic resistance rates (\%) for predominant Gram-negative bacilli recovered from tracheal aspirate of ICU patients.

\begin{tabular}{|c|c|c|c|c|}
\hline Antibiotics & Acinetobacter spp. $(N=55)$ & K. pneumoniae $(N=42)$ & Pseudomonas spp. $(N=37)$ & E. $\operatorname{coli}(N=12)$ \\
\hline Amikacin & 78.2 & 54.8 & 40.5 & 8.3 \\
\hline Ampicillin & NT & 100 & NT & 100 \\
\hline Carbenicillin & NT & NT & 43.2 & NT \\
\hline Cefepime & 96.4 & 83.3 & 78.4 & 66.7 \\
\hline Cefotaxime & 87.3 & 78.6 & 83.8 & 58.3 \\
\hline Cefoxitin & 94.5 & 81 & 89.2 & 66.7 \\
\hline Ciprofloxacin & 80 & 64.3 & 51.4 & 66.7 \\
\hline Cotrimoxazole & 96.4 & 85.7 & 86.5 & 83.3 \\
\hline Erythromycin & NT & NT & NT & 25 \\
\hline Gentamicin & 83.6 & 69 & 43.2 & 25 \\
\hline Imipenem & 23.6 & 9.5 & 16.2 & 0 \\
\hline Ofloxacin & 80 & 64.3 & 51.4 & 66.7 \\
\hline Piperacillin-tazobactam & 69.1 & 57.1 & 29.7 & NT \\
\hline Polymyxin B & 0 & 0 & 0 & NT \\
\hline
\end{tabular}

${ }^{*}$ NT: not tested.

TABLE 3: Antibiotic resistance rates (\%) for Gram-positive cocci recovered from tracheal aspirate of ICU patients.

\begin{tabular}{lcc}
\hline Antibiotics & S. aureus $(N=12)$ & S. pneumoniae $(N=1)$ \\
\hline Amikacin & $\mathrm{NT}$ & 100 \\
Ampicillin & 83.3 & 100 \\
Cefoxitin & 33.3 & 100 \\
Cefotaxime & $\mathrm{NT}$ & 100 \\
Cloxacillin & 33.3 & $\mathrm{NT}$ \\
Ciprofloxacin & 33.3 & 0 \\
Cotrimoxazole & 50 & 100 \\
Erythromycin & 41.7 & 100 \\
Gentamicin & 33.3 & 100 \\
Methicillin & 33.3 & $\mathrm{NT}$ \\
Vancomycin & 0 & 0 \\
\hline
\end{tabular}

${ }^{*}$ NT: not tested.

spp. were the most frequent ones with 22 (41.5\%) followed by $K$. pneumoniae with $13(24.5 \%)$. MBL production was confirmed in $11(36.7 \%)$ bacterial isolates and among them, 6 (54.5\%) isolates were Acinetobacter spp. followed by $K$. pneumoniae and Pseudomonas spp., each with 2 (18.2\%) and a single isolate of $K$. oxytoca $(9.1 \%)$. The results are shown in Table 4.

\section{Discussion}

The results of the study showed high growth rate, which was in accordance with the previous study, which reported culture positivity of $90 \%$ [13]. Polymicrobial growth was observed in one-tenth of the cases and the growth of multiple organisms from tracheal specimen has been mentioned in similar studies $[14,15]$. The colonization of the oropharynx, aspiration of the contaminated secretions into the lower airway, mechanical ventilation, and endotracheal tube biofilm play important role as reservoirs for infecting microorganisms [15].
In the present study, $85.4 \%$ of Acinetobacter spp., the most predominant isolate of tracheal aspirate, were MDRstrains. High level of resistance by Acinetobacter spp. was shown against cotrimoxazole (96.4\%), cefotaxime (87.3\%), ciprofloxacin $(80 \%)$, and amikacin $(78.2 \%)$. Similar trends in antimicrobial resistance (85\% to ceftazidime and ciprofloxacin, $82 \%$ to cotrimoxazole, and $67 \%$ to amikacin) of Acinetobacter spp. have been observed [16]. Acinetobacter species possess a wide array of $\beta$-lactamases that hydrolyze and confer resistance to penicillins, cephalosporins, and carbapenems. The other mechanisms of resistance include loss of porin proteins and presence of multiple efflux pumps that remove wide range of antibiotics out of the bacterial cell [17].

In this study, $73.8 \%$ of $K$. pneumoniae were MDR-strains. These isolates showed high level of resistance against cotrimoxazole $(85.7 \%)$, cefotaxime $(78.6 \%)$, gentamicin $(69 \%)$, and ciprofloxacin $(64.3 \%)$, which was in harmony with the previous study that reported resistance of $63.1 \%$ to cotrimoxazole, $90.5 \%$ to cefotaxime, $89 \%$ to gentamicin, and $65.8 \%$ to ciprofloxacin [18]. High level of drug resistance seen among $K$. pneumoniae is mediated by the production of various types of $\beta$-lactamases primarily ESBL, AmpC, and metallo$\beta$-lactamases along with drug efflux [19].

In the present study, $51.3 \%$ of Pseudomonas spp. were MDR-strains. Pseudomonas spp. were resistant to cotrimoxazole $(86 \%)$, cefotaxime $(83.8 \%)$, ciprofloxacin $(51.4 \%)$, and gentamicin $(43.2 \%)$ which was comparable to the results of two studies $[18,20]$. Pseudomonas spp. display an elevated level of drug resistance mechanisms that include production of different types of $\beta$-lactamases primarily ESBL, AmpC enzymes, and metallo-carbapenemases, aminoglycoside-modifying enzymes, loss of porin proteins, and the presence of efflux pumps like MexAB-Opr M [17].

ESBL production was confirmed in 35 (25\%) screenpositive isolates and the highest number of ESBL production was detected in Pseudomonas spp. (42.8\%) followed by $K$. pneumoniae (34.3\%), which was in contrary to one of 
TABLE 4: Profile of $\beta$-lactamase-producing bacterial strains from tracheal aspirate of ICU patients.

\begin{tabular}{|c|c|c|c|}
\hline Organisms & ESBL producers (no. and \%) & $\begin{array}{c}\text { AmpC } \beta \text {-lactamase producers } \\
(\text { no. and } \%)\end{array}$ & MBL producers (no. and \%) \\
\hline Acinetobacter spp. & $5(14.3)$ & $22(43.1)$ & $6(54.5)$ \\
\hline K. pneumoniae & $12(34.3)$ & $13(24.5)$ & $2(18.2)$ \\
\hline Pseudomonas spp. & $15(42.8)$ & $9(17.6)$ & $2(18.2)$ \\
\hline E. coli & $3(8.6)$ & $4(7.8)$ & 0 \\
\hline Enterobacter spp. & 0 & $2(4)$ & 0 \\
\hline K. oxytoca & 0 & $1(2)$ & $1(9.1)$ \\
\hline C. freundii & 0 & 0 & 0 \\
\hline P. vulgaris & 0 & 0 & 0 \\
\hline S. aureus & 0 & 0 & 0 \\
\hline S. pneumoniae & 0 & 0 & 0 \\
\hline Total & $35(25)$ & $51(37.2)$ & $11(36.7)$ \\
\hline
\end{tabular}

the studies conducted in Nepal that showed higher prevalence of E. coli with $80 \%$ and K. pneumoniae with $57.1 \%$ [21]. Higher rate of ESBL production in P. aeruginosa has now been increasingly reported due to predominantly occurring SHV- and OXA-type ESBLs [7].

AmpC $\beta$-lactamase was confirmed in $51(37.2 \%)$ of the screen-positive isolates and Acinetobacter spp. constituted 22 (43.1\%) followed by K. pneumoniae with 13 (25.5\%). Plasmidic AmpC genes are derived from the chromosomal AmpC genes of Enterobacter cloacae, Citrobacter freundii, Morganella morganii, and Hafnia alvei. Most plasmid-mediated AmpC $\beta$ lactamases are constitutively expressed, but some enzymes, such as DHA-1, DHA-2, ACT-1, CFE-1, and CMY-13, are inducible and may be more clinically dangerous conferring the capability for an organism to become more resistant during $\beta$-lactam therapy [22]. In this study, phenylboronic acid was used as an inhibitor of AmpC $\beta$-lactamase and high sensitivity and specificity of $90 \%$ and $98.2 \%$, respectively, for this method have been reported [11].

MBL production was confirmed in $11(36.7 \%)$ of the screen-positive isolates that constitute $54.5 \%$ of Acinetobacter spp., K. pneumoniae, and Pseudomonas spp., each with $18.2 \%$ and $9.1 \%$ of $K$. oxytoca. In contrast with this finding, a Korean study reported MBL production in only $14.2 \%$ of $A$. baumannii and $11.4 \%$ of $P$ aeruginosa [23]. The most common transferable MBL families include the VIM-, IMP-, GIM-, SPM-, and SIM-type enzymes, which have been detected primarily in $P$. aeruginosa but are also found in other Gramnegative bacteria, including nonfermenters and members of the family Enterobacteriaceae [24]. MBL-producing bacteria are an increasing public health problem worldwide and mortality rates have been increased due to inadequate empirical therapy [25]. In the present study, MBL production was detected using Imipenem-EDTA combined disk method, which has sensitivity and specificity of $100 \%$ and $98 \%$, respectively [26].

\section{Conclusion}

We conclude that Gram-negative bacilli were the predominant isolates of tracheal aspirate of ICU patients. There is a high rate of resistance to cephalosporins, fluoroquinolones, aminoglycosides, and cotrimoxazole. $\beta$-lactamases confer a high level of resistance to $\beta$-lactam antibiotics and these traits are usually carried in transferable genes, which are capable of being acquired by normally nonpathogenic bacteria. Therefore, early detection in routine laboratory, immediate infection control, and antibiotic stewardship programs should be implemented in order to limit the spread of $\beta$-lactamaseproducing organisms.

\section{Acknowledgment}

The authors would like to acknowledge all the staffs of National Institute of Neurological and Allied Sciences for their cooperation.

\section{References}

[1] P. Pignatti, A. Balestrino, C. Herr et al., "Tracheostomy and related host-patogen interaction are associated with airway inflammation as characterized by tracheal aspirate analysis," Respiratory Medicine, vol. 103, no. 2, pp. 201-208, 2009.

[2] J. Chastre and J.-Y. Fagon, "Ventilator-associated pneumonia," The American Journal of Respiratory and Critical Care Medicine, vol. 165, no. 7, pp. 867-903, 2002.

[3] P. Morar, V. Singh, Z. Makura et al., "Oropharyngeal carriage and lower airway colonisation/infection in 45 tracheotomised children," Thorax, vol. 57, no. 12, pp. 1015-1020, 2002.

[4] P. E. Fournier and H. Richet, "The epidemiology and control of Acinetobacter baumannii in health care facilities," Clinical Infectious Diseases, vol. 42, no. 5, pp. 692-699, 2006.

[5] E. G. Playford, J. C. Craig, and J. R. Iredell, "Carbapenemresistant Acinetobacter baumannii in intensive care unit patients: risk factors for acquisition, infection and their consequences," Journal of Hospital Infection, vol. 65, no. 3, pp. 204-211, 2007.

[6] G. A. Jacoby and L. S. M. Price, "The new beta-lactamases," The New England journal of medicine, vol. 352, no. 4, pp. 380-391, 2005.

[7] D. L. Paterson and R. A. Bonomo, "Extended-spectrum $\beta$ lactamases: a clinical update," Clinical Microbiology Reviews, vol. 18, no. 4, pp. 657-686, 2005. 
[8] B. A. Forbes, D. F. Sahm, and A. S. Weissfeld, Bailey and Scott's Diagnostic Microbiology, Mosby, 12th edition, 2007.

[9] Clinical and Laoratory Standards Institute, "Performance standards for antimicrobial susceptibility testing," 17th Informational Supplement CLSI M100-S17, Clinical and Laboratory Standards institute, Wayne, Pa, USA, 2007.

[10] A.-P. Magiorakos, A. Srinivasan, R. B. Carey et al., "Multidrugresistant, extensively drug-resistant and pandrug-resistant bacteria: an international expert proposal for interim standard definitions for acquired resistance," Clinical Microbiology and Infection, vol. 18, no. 3, pp. 268-281, 2012.

[11] P. E. Coudron, "Inhibitor-based methods for detection of plasmid-mediated AmpC $\beta$-lactamases in Klebsiella spp., Escherichia coli, and Proteus mirabilis," Journal of Clinical Microbiology, vol. 43, no. 8, pp. 4163-4167, 2005.

[12] R. C. Picão, S. S. Andrade, A. G. Nicoletti et al., "Metallo$\beta$-lactamase detection: comparative evaluation of double-disk synergy versus combined disk tests for IMP-, GIM-, SIM-, SPM-, or VIM-producing isolates," Journal of Clinical Microbiology, vol. 46, no. 6, pp. 2028-2037, 2008.

[13] P. Koirala, D. R. Bhatta, P. Ghimire, B. M. Pokhrel, and U. Devkota, "Bacteriological profile of tracheal aspirates of the patients attending a neuro-hospital of Nepal," International Journal of Advanced Life Sciences, vol. 4, pp. 60-65, 2010.

[14] E. Foglia, M. D. Meier, and A. Elward, "Ventilator-associated pneumonia in neonatal and pediatric intensive care unit patients," Clinical Microbiology Reviews, vol. 20, no. 3, pp. 409425, 2007.

[15] S. Nseir, C. D. Pompeo, P. Pronnier et al., "Nosocomial tracheobronchitis in mechanically ventilated patients: incidence, aetiology and outcome," European Respiratory Journal, vol. 20, no. 6, pp. 1483-1489, 2002.

[16] T. Reddy, T. Chopra, D. Marchaim et al., "Trends in antimicrobial resistance of Acinetobacter baumannii isolates from a Metropolitan Detroit health system," Antimicrobial Agents and Chemotherapy, vol. 54, no. 5, pp. 2235-2238, 2010.

[17] R. A. Bonomo and D. Szabo, "Mechanisms of multidrug resistance in Acinetobacter species and Pseudomonas aeruginosa," Clinical Infectious Diseases, vol. 43, no. 2, pp. S49-S56, 2006.

[18] H. B. V. Kumari, S. Nagarathna, and A. Chandramuki, "Antimicrobial resistance pattern among aerobic gram-negative bacilli of lower respiratory tract specimens of intensive care unit patients in a neurocentre," The Indian journal of chest diseases \& allied sciences, vol. 49, no. 1, pp. 19-22, 2007.

[19] T. R. Walsh, M. A. Toleman, L. Poirel, and P. Nordmann, "Metallo- $\beta$-lactamases: the quiet before the storm?" Clinical Microbiology Reviews, vol. 18, no. 2, pp. 306-325, 2005.

[20] B. V. Navaneeth and M. R. S. Belwadi, "Antibiotic resistance among gram-negative bacteria of lower respiratory tract secretions in hospitalized patients," The Indian journal of chest diseases \& allied sciences, vol. 44, no. 3, pp. 173-176, 2002.

[21] S. Poudyal, D. R. Bhatta, G. Shakya et al., "Extended spectrum â-lactamase producing multidrug resistant clinical bacterial isolates at National Public Health Laboratory, Nepal," Nepal Medical College Journal, vol. 13, no. 1, pp. 34-38, 2011.

[22] E. S. Moland, S.-Y. Kim, S. G. Hong, and K. S. Thomson, "Newer $\beta$-Lactamases: clinical and laboratory implications, part II," Clinical Microbiology Newsletter, vol. 30, no. 10, pp. 71-77, 2008.

[23] K. Lee, W. G. Lee, Y. Uh et al., "VIM- and IMP-type metallo- $\beta$ lactamase-producing Pseudomonas spp. and Acinetobacter spp. in Korean hospitals," Emerging Infectious Diseases, vol. 9, no. 7, pp. 868-871, 2003.
[24] G. Valenza, B. Joseph, J. Elias et al., "First survey of metallo$\beta$-lactamases in clinical isolates of Pseudomonas aeruginosa in a German University Hospital," Antimicrobial Agents and Chemotherapy, vol. 54, no. 8, pp. 3493-3497, 2010.

[25] C. Urban, N. Mariano, and J. J. Rahal, "In vitro double and triple bactericidal activities of doripenem, polymyxin B, and rifampin against multidrug-resistant Acinetobacter baumannii, Pseudomonas aeruginosa, Klebsiella pneumoniae, and Escherichia coli," Antimicrobial Agents and Chemotherapy, vol. 54, no. 6, pp. 2732-2734, 2010.

[26] C. Franklin, L. Liolios, and A. Y. Peleg, "Phenotypic detection of carbapenem-susceptible metallo- $\beta$-lactamase- producing gram-negative bacilli in the clinical laboratory," Journal of Clinical Microbiology, vol. 44, no. 9, pp. 3139-3144, 2006. 

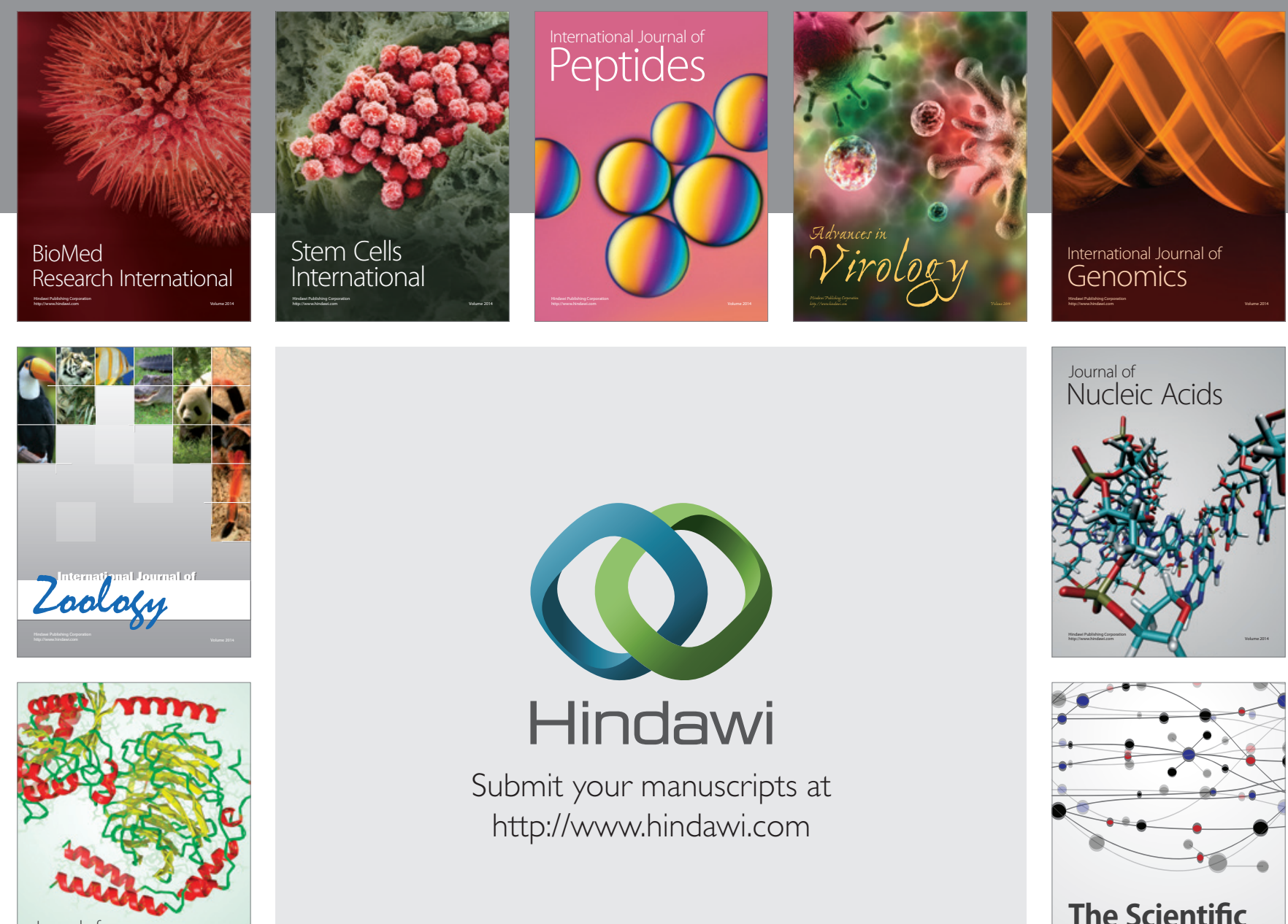

Submit your manuscripts at

http://www.hindawi.com

Journal of
Signal Transduction
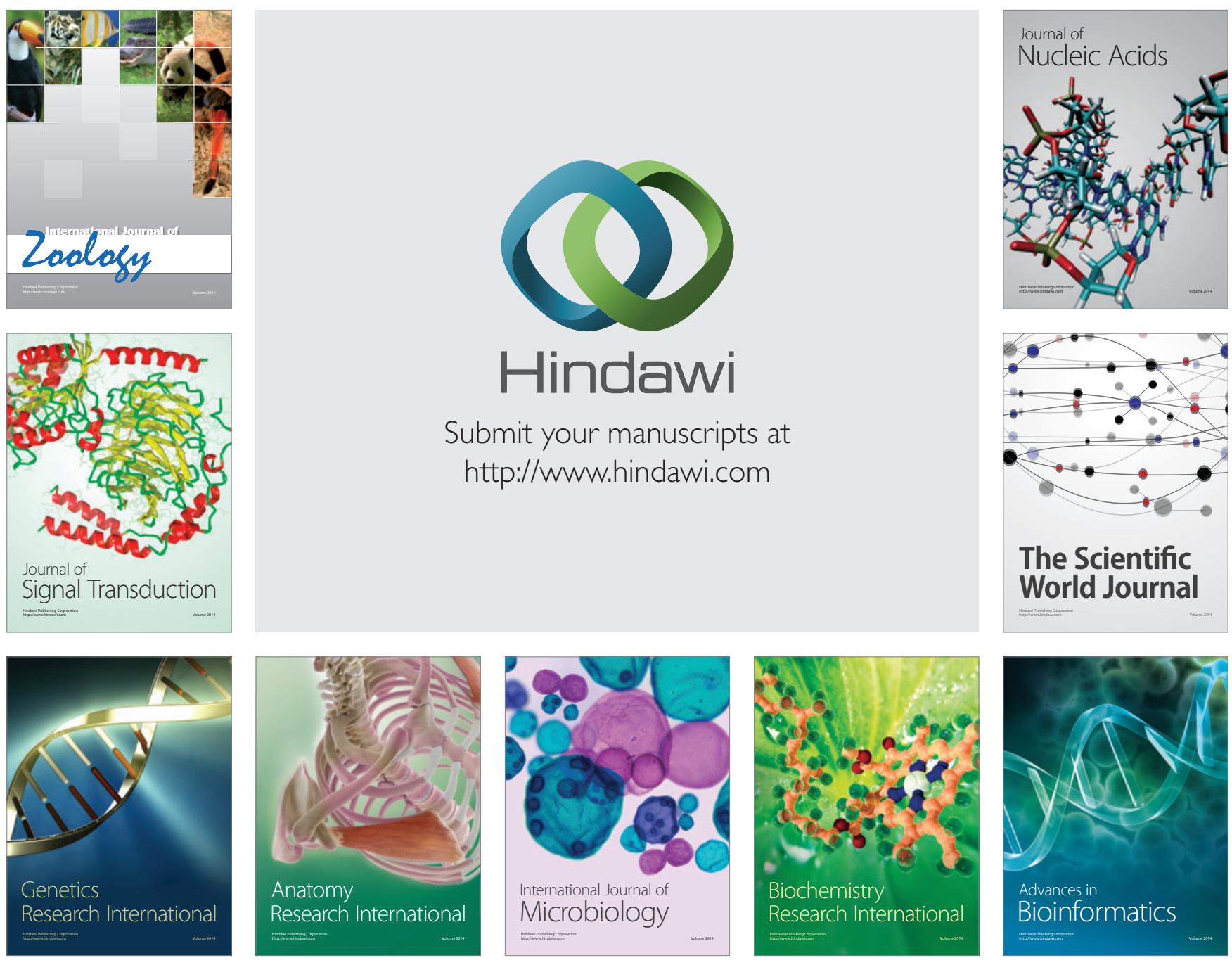

The Scientific World Journal
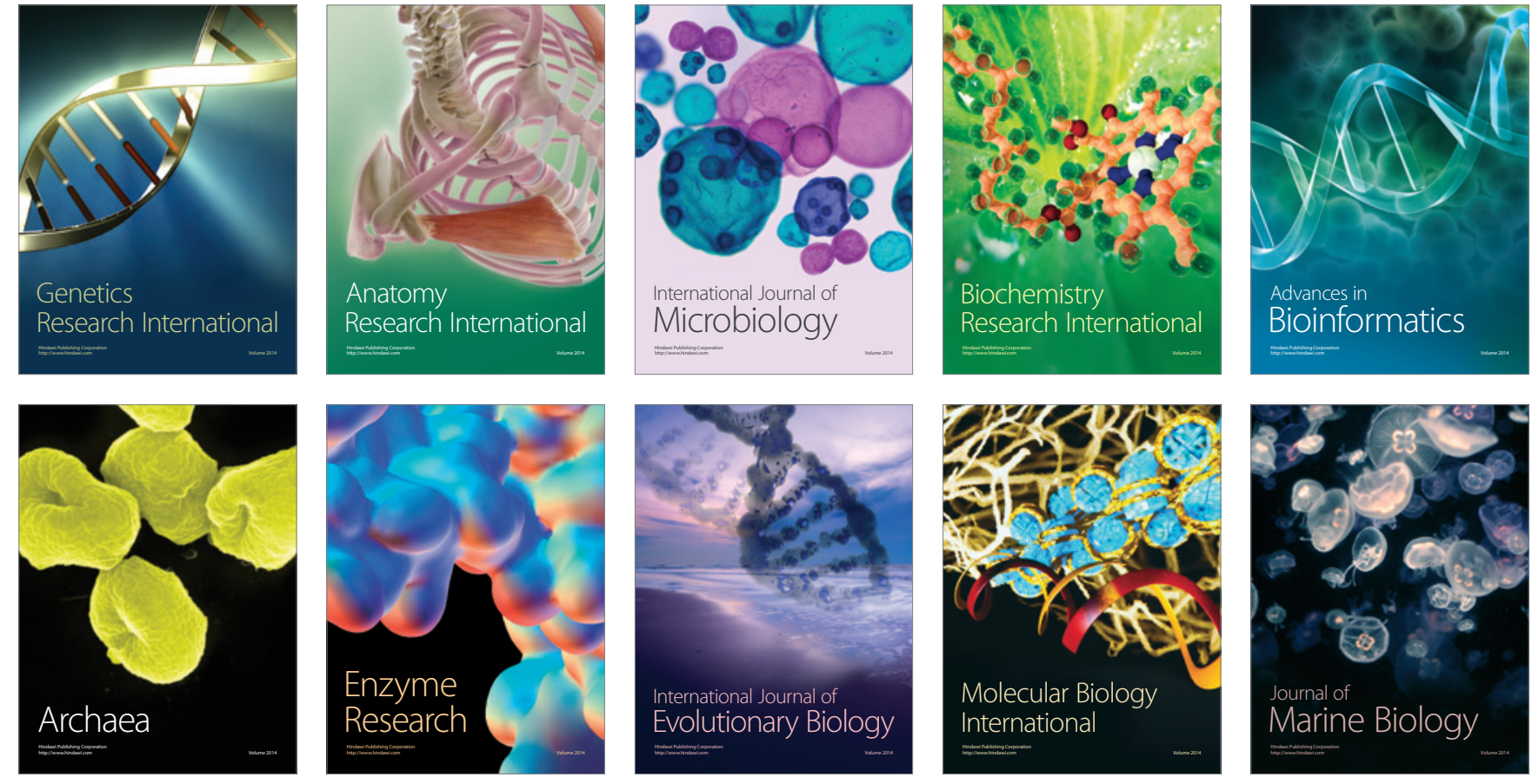